\title{
Misdiagnosis of olfactory neuroblastoma
}

\author{
Zvi R. Cohen, M.D., Eric Marmor, M.D., Gregory N. Fuller, M.D., Ph.D., \\ and Franco DeMonte, M.D.
}

Departments of Neurosurgery and Neuropathology, The University of Texas M. D. Anderson Cancer

Center, Houston, Texas

\begin{abstract}
Object. Olfactory neuroblastoma $(\mathrm{ON})$ is a rare neoplasm arising from the olfactory epithelium and found in the upper nasal cavity. The authors studied the frequency with which $\mathrm{ON}$ is misdiagnosed with other tumors of the paranasal sinuses such as neuroendocrine carcinoma (NEC), pituitary adenoma, melanoma, lymphoma, and sinonasal undifferentiated carcinoma (SNUC). Based on the belief that misdiagnosis commonly occurs, they emphasized the importance of establishing the correct diagnosis, because the treatment regimens and prognosis of these tumor types are often significantly different.

Methods. Twelve consecutive patients in whom ON was diagnosed were referred to the Department of Neurosurgery at the M. D. Anderson Cancer Center between January 1998 and March 2000.

Demographic data were collected, physical findings and mode of treatments were documented, and neuroimaging studies were assessed. Pathologists at the authors' institute reviewed the histological specimens. Only in two of 12 patients was the diagnosis of ON confirmed. Lesions in 10 patients were misdiagnosed; there were two cases of melanoma, three cases of NEC, three cases of pituitary adenoma, and two cases of SNUC. Eight of 10 patients in whom lesions were misdiagnosed required significant alteration in the initially proposed treatment plan.

Conclusions. Neurosurgeons should be acutely aware of the variety of neoplasms that occur in the paranasal region. The correct diagnosis should be ensured before initiating treatment to provide the optimum therapy and spare the patients from needless and potentially toxic treatment.
\end{abstract}

\section{KEY WORDS • olfactory neuroblastoma • immunocytochemistry • anterior skull base • paranasal sinus tumor}

Olfactory neuroblastomas are relatively uncommon tumors that originate from the olfactory epithelium in the upper nasal cavity in the region of the cribriform plate. Although some controversy persists, their primary modality of therapy is resection, usually performed by an anterior craniofacial approach and with the provision of adjuvant radiation therapy. ${ }^{29}$ In addition, recent experience suggests that there may be a therapeutic role for adjuvant chemotherapy ${ }^{4} \mathrm{~A}$ variety of other neoplastic lesions occur in the same anatomical region as ONs. Tumors such as NEC, melanoma, sinonasal lymphoma, SNUC, and pituitary adenoma can all present with similar clinical, histological, and radiological features as ON. The prognosis and therapeutic modalities for these different tumors are variable, and physicians treating neoplasms arising from the paranasal sinus region must be familiar with the diagnostic tools available to obtain a correct diagnosis before initiating therapy.

\section{CLINICAL MATERIAL AND METHODS}

Twelve consecutive patients in whom ON had been diagnosed were referred to the M. D. Anderson Cancer

Abbreviations used in this paper: $\mathrm{MR}=$ magnetic resonance; $\mathrm{NEC}=$ neuroendocrine carcinoma; $\mathrm{ON}=$ olfactory neuroblastoma; SNUC $=$ sinonasal undifferentiated carcinoma.
Center over a 15-month period between January 1998 and March 2000. The hospital records were reviewed. Demographic data, presenting symptoms, physical and imaging findings, and mode of therapy (proposed or initiated) were documented. Our neuropathologist reviewed the histopathological specimens obtained in all the patients. Immunohistochemical staining of all the specimens was performed and electron microscopy was conducted in three of them to establish a definite diagnosis.

\section{RESULTS}

In 10 patients a diagnosis of ON had been incorrectly made. Only two of the 12 lesions were ultimately confirmed to be ON. Of the 10 originally misdiagnosed neoplasms, two were confirmed to be melanoma, three to be NEC, three to be pituitary adenoma, and two to be SNUC. Of the 10 patients in whom lesions were initially misdiagnosed, seven were men and five women; their mean age at presentation was 55.9 years (range 39-79 years). Epistaxis, nasal obstruction, and headache were the most common presenting symptoms, with facial pain, decreased vision, diplopia, and proptosis occurring less frequently. As a result of misdiagnosis, eight of 10 patients required significant alteration of the treatment plan that had initially been proposed (Table 1). Two illustrative cases are described in detail, and findings in all patients are summarized in Table 1. 


\section{ILLUSTRATIVE CASES}

\section{Case 1}

This 56-year-old man initially presented to an outside institution with a history of nasal obstruction. Neuroimaging studies revealed a lesion involving the sphenoid and ethmoid sinuses. There was no evidence of bone destruction, and no intracranial disease was identified. An endoscopically guided biopsy procedure was performed and a pathological diagnosis of an $\mathrm{ON}$ was determined. Combination radiotherapy and cisplatin chemotherapy were initiated. There was no response to this therapeutic regimen, and when the patient's vision started to deteriorate, he underwent a second course of radio- and chemotherapy in which Adriamycin and vincristine were administered. Unfortunately, as a result of his treatment the patient became blind, and he developed panhypopituitarism as well as cardiomyopathy (Fig. 1). He was referred to our institution, and on review of his pathological and neuroimaging studies it was decided that a new biopsy of his lesion should be obtained. Results of this examination indicated that the lesion was in fact a pituitary adenoma.

\section{Case 8}

This 48-year-old man initially presented to an outside institution with a history of epistaxis. Neuroimaging studies revealed a lesion involving the nasal cavity and ethmoidal sinuses. There was evidence of bone destruction but no intracranial extension of the tumor was visualized. He underwent an endoscopically guided biopsy procedure, and the neoplasm was diagnosed as ON. After surgery and postoperative radiotherapy were proposed, he presented at our institution for a second opinion. Upon review, the pathologist was not satisfied that a diagnosis of ON could be made based on the original biopsy specimen, and a second biopsy procedure was performed. Analysis of this specimen revealed an NEC. Given our success in treating these tumors without surgery, ${ }^{2}$ the patient received chemotherapy and his response was excellent. He then underwent consolidating radiotherapy and his disease remains in remission, surgical intervention having been avoided (Fig. 2).

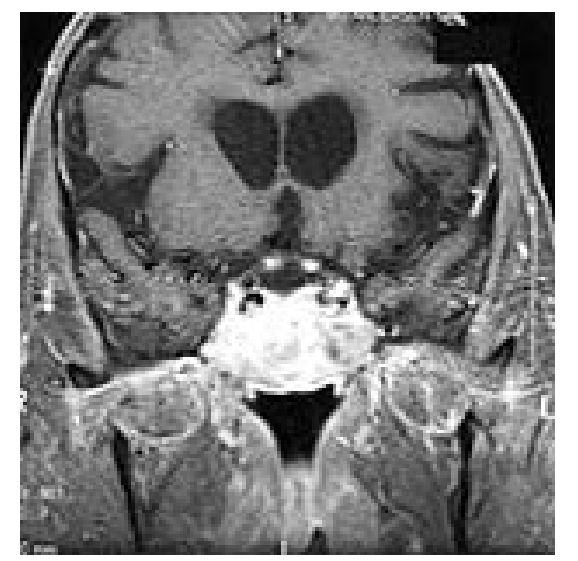

Fig. 1. Case 1. Coronal gadolinium-enhanced $\mathrm{T}_{1}$-weighted $\mathrm{MR}$ image revealing a pituitary adenoma and radiotherapy-related pathological enhancement of the optic nerves.

\section{DISCUSSION}

We have presented 12 consecutive patients in whom $\mathrm{ON}$ was initially diagnosed who were referred to the Department of Neurosurgery at the M. D. Anderson Cancer Center between January 1998 and March 2000. After careful reexamination of their diagnostic neuroimaging and pathological studies, all but two of the original diagnosis were changed. The tumors that had been initially misdiagnosed as ONs included two melanomas, three NEC, three pituitary adenomas, and two SNUC. The change in diagnosis resulted in significantly different therapeutic interventions in eight of 10 patients, with some of the patients having suffered unnecessary morbidity.

Olfactory neuroblastomas, first described by Berger and Richard in $1924^{3}$ arise from the olfactory epithelium of the nasal cavity in the region of the cribriform plate. The tumor cells are a relatively homogeneous population of small round cells set in a variable fibrillary stroma and form part of the differential diagnosis of round-cell lesions of the head and neck region. ${ }^{12}$ The presence of a fibrillary intercellular background in conjunction with the presence of Homer-Wright rosettes in an upper nasal neoplasm is considered to be diagnostic of ON. ${ }^{35}$

TABLE 1

Summary of treatment characteristics in patients in whom neoplasms were misdiagnosed as $O N^{*}$

\begin{tabular}{|c|c|c|c|c|c|}
\hline \multirow{2}{*}{$\begin{array}{l}\text { Case } \\
\text { No. }\end{array}$} & \multirow{2}{*}{$\begin{array}{c}\text { Age (yrs), } \\
\text { Sex }\end{array}$} & \multicolumn{2}{|c|}{ Treatment } & \multirow{2}{*}{$\begin{array}{c}\text { New } \\
\text { Diagnosis }\end{array}$} & \multirow[b]{2}{*}{ Final Treatment } \\
\hline & & Prior & Recommended & & \\
\hline 1 & $56, \mathrm{M}$ & biopsy, XRT, chemo & chemo & PA & observation ${ }^{\dagger}$ \\
\hline 2 & $79, \mathrm{M}$ & resection & XRT & PA & observation ${ }^{\dagger}$ \\
\hline 3 & $65, \mathrm{~F}$ & biopsy & XRT & PA & observation $^{\dagger}$ \\
\hline 4 & $52, \mathrm{M}$ & biopsy & resection, XRT & SNUC & chemo, resection, XRT \\
\hline 5 & $53, \mathrm{M}$ & resection & resection, XRT & SNUC & chemo, $\mathrm{XRT}^{\dagger}$ \\
\hline 6 & $60, \mathrm{~F}$ & biopsy & resection, XRT & NEC & chemo, XRT ${ }^{\dagger}$ \\
\hline 7 & $39, \mathrm{~F}$ & biopsy & chemo, resection & NEC & chemo, $\mathrm{XRT}^{\dagger}$ \\
\hline 8 & $48, \mathrm{M}$ & biopsy & resection, XRT & NEC & chemo, $\mathrm{XRT}^{\dagger}$ \\
\hline 9 & $68, \mathrm{M}$ & biopsy & chemo, resection, XRT & melanoma & resection, XRT \\
\hline 10 & $39, \mathrm{M}$ & resection, XRT & chemo, resection & melanoma & chemo $^{\dagger}$ \\
\hline
\end{tabular}

* Chemo $=$ chemotherapy; $\mathrm{PA}=$ pituitary adenoma; $\mathrm{XRT}=$ radiotherapy.

$\dagger$ Patients in whom a significant change in therapy was made due to reclassification of the neoplasm. 


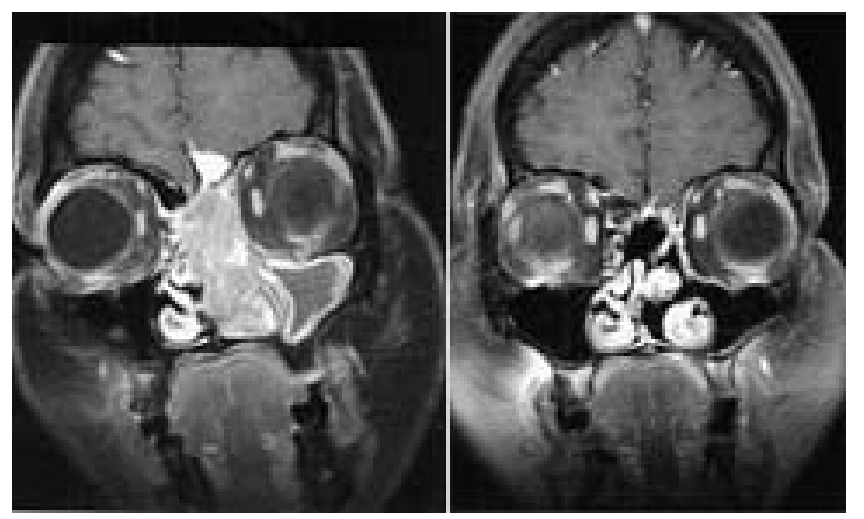

Fig. 2. Case 8. Coronal pre- (left) and posttreatment (right) gadolinium-enhanced $\mathrm{T}_{1}$-weighted MR images demonstrating complete regression of the tumor in a patient with a NEC in whom chemo- and radiotherapy were performed.

Olfactory neuroblastoma account for between 1 to $5 \%$ of malignant nasal cavity neoplasms ${ }^{39}$ and occur in all age groups with bimodal peaks in the second and fifth decades. ${ }^{38}$ Common presenting symptoms include nasal obstruction and epistaxis; facial pain, diplopia, proptosis, and anosmia are less frequently reported. ${ }^{13,29,38}$ Neuroimaging studies of ONs are nonspecific. Compared with gray matter, the signal on $\mathrm{T}_{1}$-weighted $\mathrm{MR}$ images is either hypo- or isointense. On $\mathrm{T}_{2}$-weighted MR images, the signal varies from iso- to hyperintense. Contrast enhancement is intense and of variable uniformity, with some degree of gadolinium enhancement displayed in most cases. Various staging systems $s^{5,13,23}$ have been proposed to provide better treatment guidelines and predictors of prognosis. The pathological grade of the tumor was found to be the most significant prognostic factor, with an $80 \%$ 5-year survival rate and a $40 \% 5$-year survival rate for low-grade and high-grade tumors, respectively. ${ }^{38}$

Regardless of the grade, or stage-with the possible exception of distant metastasis-surgery is the recommended mainstay of therapy in patients harboring these tumors. Multidisciplinary, aggressive craniofacial resection appears to yield the best results..$^{22,29,38}$ Surgical treatment alone is effective in cases of low-grade tumor if tumor-free margins can be obtained. The use of adjuvant radiotherapy is supported for low-grade tumors when the margins are close, for residual or recurrent disease, and for all high-grade tumors. ${ }^{38}$ Some authors have argued that radiation is best administered preoperatively, ${ }^{14,42}$ whereas others prefer to administer it postoperatively, ${ }^{13,38}$ and yet others recommend postoperative radiotherapy for Stage A tumors and preoperative radiotherapy for higher-stage lesions (Stages B and C). ${ }^{15}$ Given the rarity of ONs, it is unlikely that the optimum timing of adjuvant radiotherapy will ever be clearly determined. More recently, chemotherapy, combined with resection and radiotherapy, has been used to treat advanced-stage, high-grade, and recurrent ONs with durable remission periods. ${ }^{14,15,34}$ There are anecdotal reports of cases with unresectable lesions in which long-term survival was achieved following a combination of chemo- and radiotherapy. ${ }^{4,48}$ It appears that high-grade tumors show the greatest response to cisplatinbased chemotherapeutic treatment. ${ }^{34}$
Neuroendocrine carcinomas of the nasal cavity and paranasal sinus are exceedingly uncommon neoplasms recognized as a separate entity from ONs by both Kameya, et al., ${ }^{24}$ and Silva, et al., ${ }^{44}$ in the early 1980s. These tumors are thought to originate from the glandular epithelium of the exocrine glands found in the normal olfactory mucosa. They occur in older patients (mean age 50 years) compared with patients with $\mathrm{ON}, 2,44$ and they seldom involve the cribriform plate, confirming the hypothesis that they originate lower in the nasal vault. ${ }^{2}$ They are composed of sheets and nests of small- to intermediate-sized cells with a high nucleo/cytoplasmatic ratio, hyperchromatic nuclei, and high mitotic rates. ${ }^{40}$ The most commonly reported presenting symptoms are nasal obstruction and epistaxis. ${ }^{2,44}$ Less frequent presenting symptoms include facial pain, anosmia, and exophthalmos. ${ }^{40,44}$ These neoplasms have been shown to expand into and cause destruction of the sinuses; however, no distinctive neuroimaging characteristics have been identified on computerized tomography or MR imaging. ${ }^{25}$ Because these lesions are rare, their optimum treatment has not yet been definitively ascertained. Some investigators have advocated the use of resection in the treatment of these tumors. ${ }^{40,44}$ Our more recent experience at the M. D. Anderson Cancer Center indicates that NEC can be effectively treated using a combined regimen of chemo- and radiotherapy without the need for resection. ${ }^{2}$ The successful treatment of these tumors without surgical intervention is further supported by Chaudhry and colleagues ${ }^{8}$ who have reported a patient in whom a paranasal sinus NEC was diagnosed, who has remained disease free for 5 years after undergoing radiotherapy alone.

Sinonasal undifferentiated carcinomas were first recognized as a distinct entity from ONs by Frierson and his colleagues ${ }^{18}$ in 1986 . Consisting of medium-sized cells arranged in nests and sheets with wide trabeculae, these tumors often contain extensive necrosis and vascular permeation. ${ }^{28}$ Although few series have been reported in the literature, the epidemiology and clinical presentation for these tumors, mainly epistaxis and nasal obstruction, appear to be similar to those for ONs as well as NECs. ${ }^{18,20,28,43}$ Sinonasal undifferentiated carcinoma cannot be distinguished from other tumors of this region (with the possible exception of melanoma) based on neuroimaging features. ${ }^{41}$ In a recent study the authors suggested that the staging and grading systems commonly used for ON are also applicable for SNUC. ${ }^{37}$ The prognosis for patients with SNUC, however, is considerably less favorable than for those with $\mathrm{ON} ;{ }^{18,43}$ the overall cure rate is less than $20 \%$ and most patients die within 1.5 years of disease onset. ${ }^{26}$ Righi, et al. ${ }^{43}$ reported a mean survival of only 11.5 months following treatment. The optimum treatment for these tumors remains uncertain. Overall survival associated with SNUC is extremely poor regardless of the treatment regimens used. ${ }^{18,28,43}$ Deustch, et al., ${ }^{11}$ have recommended initial combination chemo- and radiotherapy in all cases of SNUC regardless of disease extent, followed by resection if intracranial involvement or metastatic disease are absent. Righi, et al., recommended radical resection and adjuvant therapy for patients with isolated and locally advanced disease; however, palliative chemoand radiotherapy without surgery are preferable in cases in which extensive intracranial involvement and distant 
metastatic disease are present. In a recent paper the author recommended that resection in patients with intracranial disease should not be performed until a response to adjuvant therapy has been demonstrated. ${ }^{20}$

Lymphomas arising from the paranasal sinuses are distinctly less common in Western than in Asian populations, accounting for only $0.17 \%$ of all lymphomas in the former group and $0.44 \%$ of all extranodal malignant lymphomas. ${ }^{16}$ Although virtually every subtype of lymphoma was found to occur in the paranasal sinuses in one large study ${ }^{1}$ the B-cell phenotype has a slight predominance in Western countries and the natural killer-T/NK-cell phenotype predominates in Asian and South American countries. ${ }^{47}$ Sinonasal lymphomas commonly occur in the sixth to eighth decades of life with a $2: 1$ male/female ratio. ${ }^{47}$ Patients with these tumors frequently present with nasal obstruction and facial swelling. Other less common symptoms include epistaxis, facial pain, diplopia and nasal discharge. ${ }^{19,47}$ In a recent review of the literature, Logsdon, et al., ${ }^{31}$ reported a 5-year survival rate for patients with these tumors ranging from 12 to $86 \%$. In their own large series of 70 patients, the same authors reported an overall 5-year survival rate of $52 \% .{ }^{31}$ In the past these lymphomas were treated with local radiotherapy alone with good response; however, a high incidence of local and distance recurrences were reported. ${ }^{7}$ The authors of recent reports have recommended a combination of chemotherapy and local irradiation as the optimum treatment for sinonasal lymphomas. ${ }^{31,47}$ Radiotherapy alone is considered insufficient for treatment of patients with advanced disease. ${ }^{31}$ In patients who undergo chemotherapy alone there is a higher risk of local recurrence. ${ }^{30}$ As in the case of other lymphomas, definitive resection has not been advocated as appropriate therapy for sinonasal lymphomas. ${ }^{19}$

Pituitary tumors involving the paranasal sinuses most commonly arise secondarily to invasion from an intrasellar tumor or rarely as a result of an ectopic focus. ${ }^{32}$ Ectopic pituitary adenomas are speculated to develop from ectopic cell rests that have been entrapped along the pathway of the craniopharyngeal duct during embryonic development or that have aberrantly migrated. ${ }^{21}$ These tumors share the wide histological spectrum of other pituitary adenomas, ranging from lesions with neuroendocrine features to those resembling poorly differentiated carcinomas. ${ }^{32}$ The MR imaging-documented characteristics of paranasal sinus pituitary adenomas are those of a mass, isointense to gray matter on $\mathrm{T}_{1}$-weighted sequence with a heterogeneous pattern of enhancement. ${ }^{45}$ Patients harboring these tumors may present with nasal obstruction ${ }^{10,27}$ or epistaxis. $^{32,46}$ Analysis of the literature suggests that sinonasal pituitary adenomas in general may be more aggressive than regular pituitary adenomas because many of the former are more frequently invasive macroadenomas. ${ }^{32} \mathrm{Re}-$ gardless of their pathogenesis, these tumors should be treated as regular pituitary adenomas with initial attempts at controlling prolactinomas with bromocriptine and attempts for complete resection for all other tumors. ${ }^{32}$

Sinonasal melanoma is an uncommon tumor constituting $4.8 \%$ of all neoplasms in this region. ${ }^{33}$ These neoplasms, which have migrated from the neuroectoderm, are the source of mucosal melanomas. They are composed of a variety of cells that may be round, oval, polygonal, epithelioid, or spindle shaped. ${ }^{9}$ They occur predominantly in patients in their fifth through eighth decade of life. ${ }^{17}$ The most common presenting symptoms are epistaxis and nasal obstruction. ${ }^{9,17}$ Sinonasal melanomas are aggressive tumors with a 5-year survival rate of $36 \%$ reported in a metaanalysis of 163 patients. ${ }^{6}$ Resection is the treatment of choice for these tumors. ${ }^{6,9,17,33}$ The role of adjuvant radiotherapy is more controversial and is unlikely to have more than a modest effect on survival. ${ }^{6,9}$ There is no routine role for chemotherapy in the treatment of mucosal melanoma, and this therapeutic mode should be reserved for use in randomized clinical trials. ${ }^{9}$

All of the aforementioned tumors are rarely seen, even in specialized centers. Clinical and neuroimaging features of these paranasal sinus neoplasms cannot reliably be used to establish a correct diagnosis. In fact, the clinical and neuroimaging characteristics identified in the series of misdiagnosed tumors presented in this study were all compatible with the diagnosis of $\mathrm{ON}$. The prognosis and treatment paradigms, however, vary significantly for the different types of tumors that can occur in the paranasal sinus region. As a result, it is our recommendation that in all patients presenting with paranasal sinus tumor, an adequate biopsy sample be obtained and reviewed by an experienced pathologist in collaboration with the treating physician, prior to initiation of any definitive therapy.

The pathological differentiation of these tumors can be a difficult task, even for an experienced pathologist, requiring special staining procedures and occasionally electron microscopy. Pituitary adenomas are usually cytologically bland tumors in which there are regular round nuclei, a delicate chromatin pattern, and only rare mitotic figures. Olfactory neuroblastomas are also quite homogeneous but tend to have more mitotic figures than pituitary adenomas. Homer-Wright rosettes are not invariably seen, but their presence can aid in making the diagnosis of ON. Sinonasal undifferentiated carcinoma, NEC, lymphomas, and melanomas are all generally high-grade tumors with frequent mitotic figures and pleomorphic nuclei. A battery of immunohistochemical stains is widely available and is indispensable for characterizing tumors arising from the paranasal sinus region. The staining characteristics of these tumors are summarized in Table 2. Some of the staining characteristics of ON, NEC, and SNUC are presented in Fig. 3. Of all the paranasal sinus tumors, it is most difficult to differentiate ON from NEC, and despite the usefulness of immunohistochemistry, electron microscopy may be required to distinguish these two neo-

\section{TABLE 2}

Characteristics of immunohistochemical testing of paranasal sinus tumors*

\begin{tabular}{lccccc}
\hline \hline \multicolumn{1}{c}{ Tumor Type } & $\begin{array}{c}\text { Neuronal } \\
\text { Markers }\end{array}$ & Keratin & $\begin{array}{c}\text { Pituitary } \\
\text { Hormones }\end{array}$ & $\begin{array}{c}\text { S-100, } \\
\text { HMB-45 }\end{array}$ & $\begin{array}{c}\text { CD45 } \\
\text { (LCA) }\end{array}$ \\
\hline ON & + & - & - & - & - \\
SNUC & - & + & - & - & - \\
NEC & + & + & - & - & - \\
pituitary adenoma & + & + & +- & - & - \\
melanoma & - & - & - & + & - \\
lymphoma & - & - & - & - & + \\
\hline
\end{tabular}

$*+=$ frequent presence of staining; $-=$ frequent absence of staining; $+/-=$ staining variably present. 


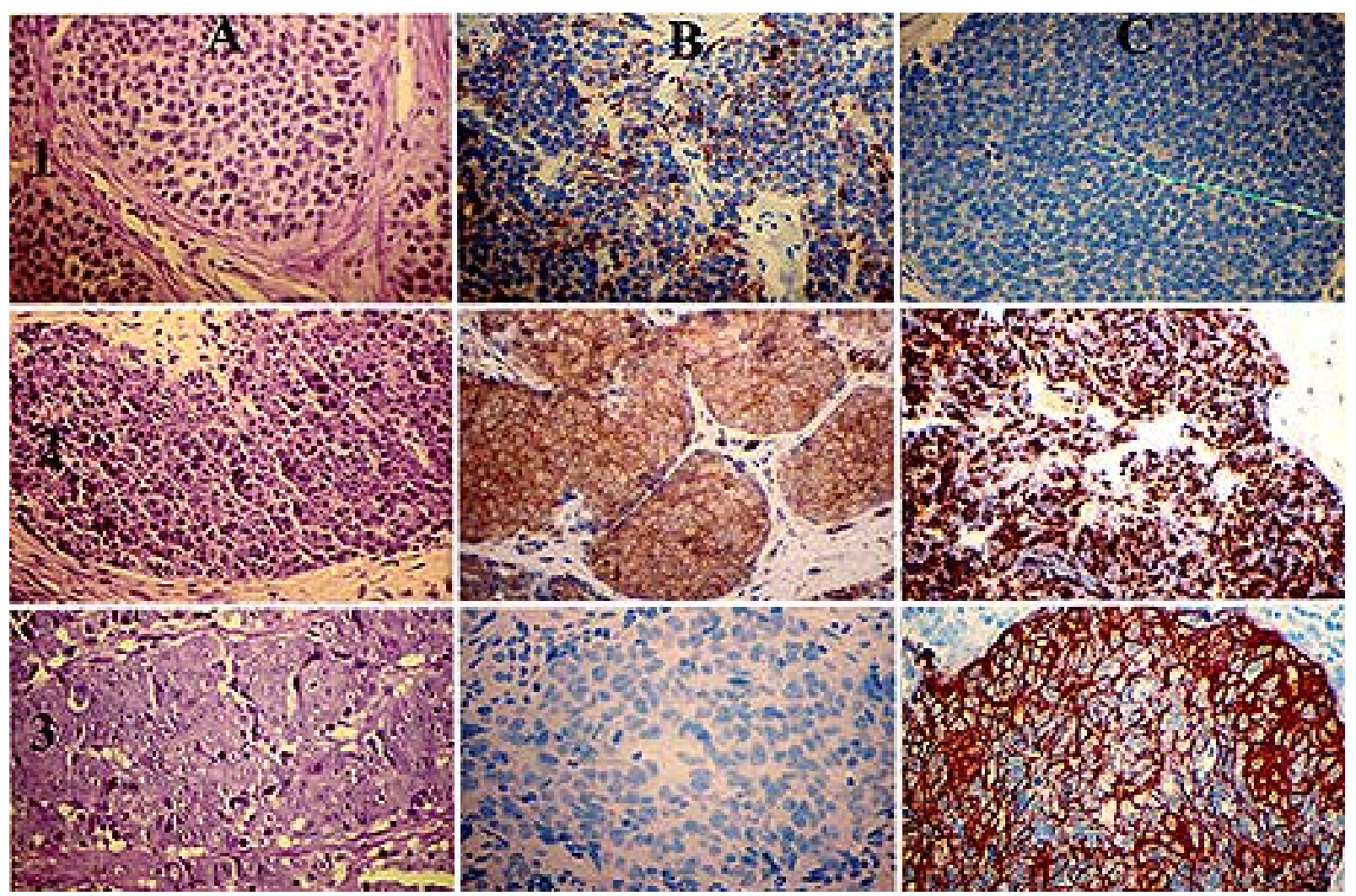

Fig. 3. Photomicrographs. Column A): H \& E; Column B): synaptophysin; and Column C): cytokeratin. Row 1: an ON. Row 2: an NEC. Row 3: an SNUC. The stains for synaptophysin are positive in both the ON and the NEC but negative in the SNUC. Cytokeratin staining is negative in the ON but positive in both NEC and SNUC.

plasms. ${ }^{36,44}$ Ultrastructurally, ONs consist of blastic cells with neuroendocrine granules, dendritic processes, and nerve endings with synaptic structures, whereas NEC have rare neuroendocrine granules, simple cytoplasmic borders, and, most importantly, no dendritic processes.

Moreover, electron microscopy is useful in the diagnostic categorization of other sinonasal tumors as well. Electron microscopy studies of SNUC show ultrastructural characteristics of closely apposed cell membranes with occasional attachment cell junctions; however, they do not have dense core granules, dendritic process, or synaptic structures. Lymphomas are characterized by the absence of cell attachment junctions, whereas melanomas have the ultrastructurally diagnostic appearance of melanin granules. ${ }^{36}$

In summary we recommend the use of immunohistochemical and electron microscopy studies to differentiate between the variety of round-cell neoplasms arising in the sinonasal region to establish the accurate diagnosis, which is vital for providing the optimum treatment for patients.

\section{CONCLUSIONS}

Tumors arising from the paranasal sinus region are relatively rare. Clinicians treating patients with these tumors must be aware of the spectrum of neoplasms that occur in this region. Definitive treatment of these tumors should not be initiated until a thorough neuroimaging examination and subsequent biopsy sampling are undertaken. A spectrum of immunohistological tools is available to help distinguish the often otherwise similar pathological features that these tumors share. Furthermore, in some instances electron microscopy may be necessary to establish the diagnosis. The treatment regimens for these tumor types are often significantly different from the accepted treatment of ONs. Ensuring that a correct diagnosis is made before starting treatment will spare the patient from needless and potentially toxic therapy.

\section{References}

1. Abbondanzo SL, Wenig BM: Non-Hodgkin's lymphoma of the sinonasal tract. A clinicopathologic and immunophenotypic study of 120 cases. Cancer 75:1281-1291, 1995

2. Austin JR, Cebrun H, Kershisnik MM, et al: Olfactory neuroblastoma and neuroendocrine carcinoma of the anterior skull base: treatment results at M. D. Anderson Cancer Center. Skull Base Surg 6:1-8, 1996

3. Berger L, Luc G, Richard D: L'esthesioneuroepitheliome olfactif. Bull Assoc Fr Etude Cancer 13:410-421, 1924 (Reference unverified)

4. Bhattacharyya N, Thornton AF, Joseph MP, et al: Successful treatment of esthesioneuroblastoma and neuroendocrine carcinoma with combined chemotherapy and proton radiation. Results in 9 cases. Arch Otolaryngol Head Neck Surg 123: 34-40, 1997

5. Biller HF, Lawson W, Sachdev VP, et al: Esthesioneuroblastoma: surgical treatment without radiation. Laryngoscope 100: 1199-1201, 1990 
6. Brandwein MS, Rothstein A, Lawson W, et al: Sinonasal melanoma. A clinicopathologic study of 25 cases and literature meta-analysis. Arch Otolaryngol Head Neck Surg 123: 290-296, 1997

7. Burton GV, Atwater S, Borowitz MJ, et al: Extranodal head and neck lymphoma. Prognosis and patterns of recurrence. Arch Otolaryngol Head Neck Surg 116:69-73, 1990

8. Chaudhry MR, Akhtar S, Kim DS: Neuroendocrine carcinoma of the ethmoid sinus. Eur Arch Otorhinolaryngol 251: 461-463, 1994

9. De Meerleer GO, Vermeersch H, van Eijkeren M, et al: Primary sinonasal mucosal melanoma: three different therapeutic approaches to inoperable local disease or recurrence and a review of the literature. Melanoma Res 8:449-457, 1998

10. Dent JA, Rickhuss PK: Invasive pituitary adenoma presenting with nasal obstruction. J Laryngol Otol 103:605-609, 1989

11. Deutsch BD, Levine PA, Stewart FM, et al: Sinonasal undifferentiated carcinoma: a ray of hope. Otolaryngol Head Neck Surg 108:697-700, 1993

12. Devaney K, Wenig BM, Abbondanzo SL: Olfactory neuroblastoma and other round cell lesions of the sinonasal region. Mod Pathol 9:658-663, 1996

13. Dulguerov P, Calcaterra T: Esthesioneuroblastoma: the UCLA experience 1970-1990. Laryngoscope 102:843-849, 1992

14. Eden BV, Debo RF, Larner JM, et al: Esthesioneuroblastoma. Long-term outcome and patterns of failure-the University of Virginia experience. Cancer 73:2556-2562, 1994

15. Eriksen JG, Bastholt L, Krogdahl AS, et al: Esthesioneuroblastoma-what is the optimal treatment? Acta Oncol 39:231-235, 2000

16. Fellbaum C, Hansmann ML, Lennert K: Malignant lymphomas of the nasal cavity and paranasal sinuses. Virchows Arch A Pathol Anat Histopathol 414:399-405, 1989

17. Freedman HM, DeSanto LW, Devine KD, et al: Malignant melanoma of the nasal cavity and paranasal sinuses. Arch Otolaryngol 97:322-325, 1973

18. Frierson HF Jr, Mills SE, Fechner RE, et al: Sinonasal undifferentiated carcinoma. An aggressive neoplasm derived from schneiderian epithelium and distinct from olfactory neuroblastoma. Am J Surg Pathol 10:771-779, 1986

19. Frierson HF Jr, Mills SE, Innes DJ Jr: Non-Hodgkin's lymphomas of the sinonasal region: histologic subtypes and their clinicopathologic features. Am J Clin Pathol 81:721-727, 1984

20. Gorelick J, Ross D, Marentette L, et al: Sinonasal undifferentiated carcinoma: case series and review of the literature. Neurosurgery 47:750-755, 2000

21. Hori A: Suprasellar peri-infundibular ectopic adenohypophysis in fetal and adult brains. J Neurosurg 63:113-115, 1985

22. Irish J, Dasgupta R, Freeman J, et al: Outcome and analysis of the surgical management of esthesioneuroblastoma. J Otolaryngol 26:1-7, 1997

23. Kadish S, Goodman M, Wang CC: Olfactory neuroblastoma. A clinical analysis of 17 cases. Cancer 37:1571-1576, 1976

24. Kameya T, Shimosato Y, Adachi I, et al: Neuroendocrine carcinoma of the paranasal sinus: a morphological and endocrinological study. Cancer 45:330-339, 1980

25. Kanamalla US, Kesava PP, McGuff HS: Imaging of nonlaryngeal neuroendocrine carcinoma. AJNR 21:775-778, 2000

26. Kerrebijn JD, Tietze L, Mock D, et al: Sinonasal undifferentiated carcinoma. J Otolaryngol 27:40-42, 1998

27. Kikuchi K, Kowada M, Sasaki J, et al: Large pituitary adenoma of the sphenoid sinus and the nasopharynx: report of a case with ultrastructural evaluations. Surg Neurol 42:330-334, 1994

28. Levine PA, Frierson HF Jr, Stewart FM, et al: Sinonasal undifferentiated carcinoma: a distinctive and highly aggressive neoplasm. Laryngoscope 97:905-908, 1987

29. Levine PA, Gallagher R, Cantrell RW: Esthesioneuroblastoma: reflections of a 21-year experience. Laryngoscope 109: $1539-1543,1999$
30. Liang R, Todd D, Chan TK, et al: Treatment outcome and prognostic factors for primary nasal lymphoma. J Clin Oncol 13:666-670, 1995

31. Logsdon MD, Ha CS, Kavadi VS, et al: Lymphoma of the nasal cavity and paranasal sinuses: improved outcome and altered prognostic factors with combined modality therapy. Cancer 80:477-488, 1997

32. Luk IS, Chan JK, Chow SM, et al: Pituitary adenoma presenting as sinonasal tumor: pitfalls in diagnosis. Hum Pathol 27: 605-609, 1996

33. Matias C, Corde J, Soares J: Primary malignant melanoma of the nasal cavity: a clinicopathologic study of nine cases. J Surg Oncol 39:29-32, 1988

34. McElroy EA Jr, Buckner JC, Lewis JE: Chemotherapy for advanced esthesioneuroblastoma: the Mayo Clinic experience. Neurosurgery 42:1023-1028, 1998

35. Mills SE, Frierson HF Jr: Olfactory neuroblastoma. A clinicopathologic study of 21 cases. Am J Surg Pathol 9:317-327, 1985

36. Min KW: Usefulness of electron microscopy in the diagnosis of "small" round cell tumors of the sinonasal region. Ultrastruct Pathol 19:347-363, 1995

37. Miyamoto RC, Gleich LL, Biddinger PW, et al: Esthesioneuroblastoma and sinonasal undifferentiated carcinoma: impact of histological grading and clinical staging on survival and prognosis. Laryngoscope 110:1262-1265, 2000

38. Morita A, Ebersold MJ, Olsen KD, et al: Esthesioneuroblastoma: prognosis and management. Neurosurgery 32:706-715, 1993

39. O'Connor TA, McLean P, Juillard GJ, et al: Olfactory neuroblastoma. Cancer 63:2426-2428, 1989

40. Perez-Ordonez B, Caruana SM, Huvos AG, et al: Small cell neuroendocrine carcinoma of the nasal cavity and paranasal sinuses. Hum Pathol 29:826-832, 1998

41. Phillips CD, Futterer SF, Lipper MH, et al: Sinonasal undifferentiated carcinoma: CT and MR imaging of an uncommon neoplasm of the nasal cavity. Radiology 202:477-480, 1997

42. Polin RS, Sheehan JP, Chenelle AG, et al: The role of preoperative adjuvant treatment in the management of esthesioneuroblastoma: the University of Virginia experience. Neurosurgery 42:1029-1037, 1998

43. Righi PD, Francis F, Aron BS, et al: Sinonasal undifferentiated carcinoma: a 10-year experience. Am J Otolaryngol 17: 167-171, 1996

44. Silva EG, Butler JJ, Mackay B, et al: Neuroblastomas and neuroendocrine carcinomas of the nasal cavity: a proposed new classification. Cancer 50:2388-2405, 1982

45. Slonim SM, Haykal HA, Cushing GW, et al: MRI appearances of an ectopic pituitary adenoma: case report and review of the literature. Neuroradiology 35:546-548, 1993

46. Tovi F, Hirsch M, Sacks M, et al: Ectopic pituitary adenoma of the sphenoid sinus: report of a case and review of the literature. Head Neck 12:264-268, 1990

47. Vidal RW, Devaney K, Ferlito A, et al: Sinonasal malignant lymphomas: a distinct clinicopathological category. Ann Otol Rhinol Laryngol 108:411-419, 1999

48. Zappia JJ, Carroll WR, Wolf GT, et al: Olfactory neuroblastoma: the results of modern treatment approaches at the University of Michigan. Head Neck 15:190-196, 1993

Manuscript received March 18, 2002.

Accepted in final form April 15, 2002.

Address reprint requests to: Franco DeMonte, M.D., Department of Neurosurgery, The University of Texas, M. D. Anderson Cancer Center, 1515 Holcombe Boulevard, Box 442, Houston, Texas 77030 